\title{
Stereotyp und Ideologie. Marginalisierung kritischer Forschung in der Kommunikations- wissenschaft
}

Martina Thiele

Keywords: Stereotyp, Ideologie, Fachgeschichte, Metaanalyse, Phasen

\section{Abstract}

Der Beitrag verfolgt zwei Ziele: Zum einen möchte er den Zusammenhang zwischen Stereotypen und Ideologie verdeutlichen, zum anderen Phasen des Aufschwungs und der Marginalisierung kritischer Stereotypenforschung in der deutschen Kommunikationswissenschaft nachzeichnen. Grundlage des Phasenmodells sind Metaanalysen der Forschung zu Stereotypen. Sie führen auch zur Klärung wissenschaftstheoretischer Positionen. Die Marginalisierung (ideologie-)kritischer Positionen wird abschließend durch ein Beispiel - Reaktionen auf das Werk Franz Dröges veranschaulicht.

Thiele, Martina. 2019. „Stereotyp und Ideologie. Marginalisierung kritischer Forschung in der Kommunikationswissenschaft". In Ideologie, Kritik, Öffentlichkeit. Verhandlungen des Netzwerks Kritische Kommunikationswissenschaft, herausgegeben von Uwe Krüger und Sebastian Sevignani, 290-318. Universität Leipzig. DOI: 10.36730/ideologiekritik.2019.14

Martina Thiele | Universität Salzburg | martina.thiele@sbg.ac.at 


\section{Stereotype und Stereotypenforschung}

Im Verlauf des 20. Jahrhunderts haben sich Forscher*innen verschiedener wissenschaftlicher Disziplinen mit der Entstehung und mit möglichen individuellen wie gesellschaftlichen Funktionen von Stereotypen, Feindbildern, Vorurteilen und Klischees beschäftigt. Als ein Klassiker gilt Walter Lippmanns Public Opinion von 1922, ein Werk, das erst 1964 auf Deutsch erschienen ist (Lippmann 1922; Lippmann 1964). ${ }^{1}$ Lippmann, der Public Opinion unter dem Eindruck der im Ersten Weltkrieg verfeinerten Propagandatechniken verfasst hat, definiert Stereotype als „pictures in our heads" und spricht damit ihre kognitive Dimension an. Zugleich beschreibt er aber auch die politischen und sozialen Folgen stereotyper Wahrnehmungs- und Berichterstattungsmuster und ihre positiven wie negativen Funktionen.

Laut Jürgen Wilke $(2007,603)$ hat Lippmann den Begriff des Stereotyps „wenn nicht eingeführt, so doch in Umlauf gebracht, noch bevor die sozialpsychologische Stereotypen- und Vorurteilsforschung richtig einsetzte“. Nicht nur aus der Sozialpsychologie, sondern auch der Linguistik, der Politik- und der Geschichtswissenschaft kamen in der zweiten Hälfte des 20. Jahrhunderts wichtige Impulse. So haben etwa die Forscher*innen um Theodor W. Adorno in The Authoritarian Personality (Adorno, FrenkelBrunswik, Levinson und Sanford 1950) und später am wiedergegründeten Frankfurter Institut für Sozialforschung theoretische wie empirische Grundlagen für die Stereotypen- und Vorurteilsforschung geschaffen. Die Kommunikations- und Medienwissenschaft hat diese Anregungen zum Teil aufgegriffen, zum Teil aber auch übersehen bzw. nicht zur Kenntnis nehmen wollen. Andererseits sind in den genannten Nachbardisziplinen (Massen-)Medien als Konstrukteure und Vermittler von Stereotypen überwiegend nicht berücksichtigt worden.

Aufschlussreich ist, wie sich im Laufe der Jahrzehnte und je nach akademischer Disziplin sowie theoretischer Positionierung Stereotyp-

1 Der Berliner Publizistikwissenschaftler Fritz Eberhardt hat das Werk 1964 für die Wochenzeitung Die Zeit rezensiert und die mangelhafte Übersetzung kritisiert (Eberhardt 1964). 2018 ist eine Neuübersetzung erschienen. Die Herausgeber*innen Walter Otto Ötsch und Silja Graupe führen in das Werk Lippmanns ein und meinen, es sei höchste Zeit, sich das Wissen über die Macht innerer Bilder und die Manipulation öffentlicher Meinung wieder anzueignen, das vor fast einhundert Jahren bereits vorlag (vgl. Ötsch und Graupe 2018, 53). 
definitionen verändert haben. Eine weitere, viel zitierte Definition stammt neben der Walter Lippmanns von der Linguistin Uta Quasthoff. Sie verweist $u$. a. auf die ungerechtfertigte Pauschalisierung, die mit Stereotypisierung einhergeht: „Ein Stereotyp ist der verbale Ausdruck einer auf soziale Gruppen oder einzelne Personen als deren Mitglieder gerichteten Überzeugung. Es hat die logische Form eines Urteils, das in ungerechtfertigt vereinfachender und generalisierender Weise, mit emotional-wertender Tendenz, einer Klasse von Personen bestimmte Eigenschaften oder Verhaltensweisen zu- oder abspricht. Linguistisch ist es als Satz beschreibbar" (Quasthoff 1973, 28).

Ihre Definition ist auch aus kommunikationswissenschaftlicher Sicht hilfreich, weil Quasthoff die "Bilder in unseren Köpfen" als etwas Beschreibbares versteht. Ein Stereotyp lässt sich in Worte fassen, unabhängig davon, ob es medial in Form eines Bildes (Karikatur, Foto), in Form einer Bildsequenz oder eines Textes vermittelt worden ist.

Sozialpsychologische Definitionen beschreiben Stereotype vor allem als Kognitionen oder auch als „Korrelationen zwischen Merkmalen und einer (sozialen) Kategorie“ (Six und Six-Materna 2006, 325). Auf ein Werturteil wird meist verzichtet, ebenso auf die Thematisierung einer Unangemessenheit bzw. Realitätsinadäquanz von Stereotypen.

Meine Definition versucht verschiedene Aspekte zu integrieren: Stereotype beruhen auf Kategorisierung und Attribuierung. Bestimmte Eigenschaften werden als eng verbunden mit zuvor kategorisierten Personen(gruppen), Gegenständen, Situationen oder auch abstrakten Dingen und Ideen wahrgenommen und diese Wahrnehmungen dann als Behauptungen formuliert, in Medien verbalisiert und visualisiert. Dass es sich bei jenen Behauptungen um nichtzutreffende, auf Fehlwahrnehmungen beruhende Aussagen handeln könnte, ist hier impliziert. Doch geht es nicht nur um richtige oder falsche Attribuierungen. Der vorgelagerte Prozess der Kategorisierung bzw. das Denken in Kategorien und Dualismen ist m. E. und gerade auch mit Blick auf Ideologien wie Rassismus, Klassismus und Sexismus entscheidender als die Zuschreibung von Eigenschaften.

$\mathrm{Zu}$ Stereotypdefinitionen ist noch anzumerken, dass sie kaum alle mit Stereotypisierung, ihren Ursachen sowie Folgen verbundenen Aspekte berücksichtigen können, denn Definitionen sollen vor allem kurz und Ausgangspunkt weiterer Forschung sein. Doch fällt auf, dass Stereotypdefinitionen in kommunikationswissenschaftlichen Studien häufig gar 
nicht vorgenommen oder aber dadurch ersetzt werden, dass die Autor*innen soziale wie individuelle Funktionen von Stereotypen aufzählen und meinen, damit hinreichend erklärt zu haben, was Stereotype sind. Umgangen wird so eine tiefergehende theoretische Befassung mit Stereotypen, die zu einer Positionierung in den Debatten führen würde, die in der Stereotypenforschung anhaltend sind und die an anderer Stelle als "epistemologische Herausforderungen" (Thiele 2015) schon ausführlich beschrieben wurden. Es geht in diesen Theoriedebatten beispielsweise um das Verhältnis von Stereotypen, Realität und Medienrealität, die sogenannte "kernel-of-truth-debate", um Attribuierungen und die Frage, ob es nicht auch positive Stereotype gibt, um (De-)Kategorisierung und Intersektionalität (Thiele 2017; 2019), um Stereotypenbeständigkeit oder -wandel und selbstverständlich auch um Funktionen von Stereotypen, dann aber auch weitergehend um eine Kritik des Funktionalismus in der kommunikationswissenschaftlichen Forschung. Denn tendenziell blendet eine funktionalistische Sicht auf Gesellschaft Fragen der Macht und des Machterhalts aus. Sie zielt auf den Erhalt des Status quo und ist damit keineswegs unpolitisch, wie auch Dennis McQuail (1994, 78) betont: "While apolitical in formulation, it suits pluralist and voluntarist conceptions of the fundamental mechanisms of social life and has a conservative bias to the extent that the media are likely to be seen as a means of maintaining society as it is rather than a source of major change."

Eingedenk dieser kritischen Perspektive auf funktionalistische Ansätze widme ich mich im folgenden Abschnitt einer Funktion bzw. Dysfunktion von Stereotypen, die, wie ich vermute, aus ideologischen Gründen in der kommunikationswissenschaftlichen Literatur nur selten thematisiert wird: der ideologischen Funktion von Stereotypen.

\section{Stereotype und Ideologie}

Wenn es um Stereotype und ihre Funktionen geht, wird zumeist als erste die der Komplexitätsreduktion angeführt. Aussagen wie "Stereotype sind unvermeidlich, weil sie ,automatisch' aktiviert werden“ und "Stereotype erleichtern die Orientierung in einer komplexen Welt" werden durch Ergebnisse der Kognitionsforschung gestützt und scheinen mehrheitlich akzeptiert. Eine auf Selektionen und Generalisierungen von Informationen basierende Kommunikation gilt als funktional und „effizient". 
In der Literatur sind so gut wie keine kritischen Auseinandersetzungen mit dieser "rationalen", "ökonomischen" Sicht zu finden, obwohl sie als ebenfalls stereotyp, als ideologisch und als Zeichen für "cognitive laziness“", für Denkfaulheit, gedeutet werden könnte. Schließlich verdecken Stereotype und Vorurteile, wie komplex die Welt nun einmal ist.

In den Blick genommen werden sollten jedenfalls Funktionen wie Dysfunktionen von Stereotypen, denn sie können, je nachdem, ob es um das Individuum oder die soziale Gruppe geht, ob jemand zur Ingroup oder zur Outgroup gehört, stereotypisierende oder stereotypisierte Person ist, positive oder negative Folgen haben. Das ist sofort nachvollziehbar bei Stereotyp-Funktionen wie Orientierung, Wissen, Identitätsbildung und -stabilisierung, Integration und Desintegration.

Doch was ist mit der Ideologiefunktion? Thematisiert wird sie nur von einigen Autor*innen, die sie auch die politische oder propagandistische Funktion von Stereotypen nennen. So befasst sich Franz Dröge in einem Abschnitt seiner Studie Publizistik und Vorurteil (1967) mit "Stereotype und Ideologie", ohne aber auf den Ideologiebegriff näher einzugehen. Insbesondere in Krisenzeiten, wenn die alten Gewissheiten erschüttert werden, erhielten „universelle Lösungen (ideologische Propaganda) den Vorzug vor rationaler, aufklärerischer Wirklichkeitsbewältigung“ (Dröge 1967, 162f.). Eine politisch-ideologische Funktion von Stereotypen ist nach dieser Interpretation eng verbunden mit den Funktionen der Orientierung und Stabilisierung des Selbst. Dröge verweist auf das „Gewißheitsbedürfnis" und die „Intoleranz für Vieldeutigkeit“ bzw. „Ablehnung von Unbestimmtheit", die bei "autoritären Persönlichkeiten“ (vgl. Adorno, Frenkel-Brunswik, Levinson und Sanford 1950) noch stärker ausgeprägt seien. Als Beleg für „ein Interdependenzverhältnis zwischen Stereotype und Ideologie einerseits und dem Bedürfnis nach subjektiver Gewißheit, das die Intoleranz für Vieldeutigkeit motiviert, andererseits" (Dröge 1967,163) führt er die Situation in Deutschland nach Ende des Ersten Weltkriegs an und argumentiert damit ähnlich wie Walter Lippmann, dessen Auseinandersetzung mit Stereotypen in Public Opinion (1922) ebenfalls Folge der Erfahrung des Ersten Weltkriegs ist.

Dass Stereotype Hochkonjunktur in Krisenzeiten hätten und in Umbruchszeiten eine besondere Ideologieanfälligkeit $\mathrm{zu}$ konstatieren sei, sind in der Literatur häufig anzutreffende Erklärungen. Ihre empirische Überprüfung ist allerdings schwierig, schon die Bestimmung von „Krise“ oder "Umbruchszeit" fällt nicht eindeutig aus. Dennoch wird ein 
Zusammenhang zwischen politisch unsicherer Lage und Stereotypenhäufigkeit, -verbreitung und -verfestigung hergestellt. Dröge gelangt zu dem Schluss: „Allen Ideologien liegen also Stereotypen zugrunde, weil dies ein Grundzug der Ideologiebildung ist." (Dröge 1967, 169)

Was aber meint "Ideologie“ und worin genau besteht die ideologische Funktion von Stereotypen? Mit dem Ideologiebegriff aus politikwissenschaftlicher Sicht setzen sich Autor*innen im von Anton Pelinka 1981 herausgegebenen Band Ideologien im Bezugsfeld von Geschichte und Gesellschaft auseinander. Pelinka geht einleitend auf die Vielschichtigkeit des Ideologiebegriffs ein und unterscheidet einen Neutralität beanspruchenden Ideologiebegriff deutlich vom in der Tradition des Marxismus stehenden (vgl. Pelinka 1981, 9). Auch Kurt Lenk (1981, 98f.) trifft diese Unterscheidung zwischen wissenssoziologischen Positionen, wie sie etwa Karl Mannheim vertreten hat, und marxistischen. Sehen Vertreter*innen wissenssoziologischer Positionen in jeder Auslegung von menschlich-historischem Selbstverständnis Ideologie, ist Ideologie nach Karl Marx und Friedrich Engels gesellschaftlich erzeugtes falsches Bewusstsein ${ }^{2}$ und Ausdruck ökonomischer und politischer Gegebenheiten. Sie ergibt sich aus der unterschiedlichen Stellung der Individuen im Produktionsprozess. Wenn auch ihre Inhalte sich wandelten, sei doch ihre Funktion die gleiche: Erhaltung des Status quo und somit der bestehenden Machtverhältnisse im Kapitalismus. Den Ideologiebegriff entwickeln Marx und Engels in verschiedenen Schriften, so in der Deutschen Ideologie (vgl. MEW 1958, Bd. 3, 26f.), in der im Vorwort von Zur Kritik der Politischen Ökonomie skizzierten Basis-Überbau-Lehre, wo von den „ideologischen Formen“ (MEW 1958, Bd. 13, 8f.) die Rede ist, in der Auseinandersetzung mit gegnerischen Strömungen innerhalb der Arbeiterbewegung und in den Engelsschen Altersbriefen. Hans-Christoph Rauh (1969) extrahiert aus den verschiedenen Verwendungen des Ideologie-Begriffs bei Marx und Engels eine Definition, die vor allem durch die Camera-Obscura-Metapher in der Deutschen Ideologie geprägt scheint. Danach ist Ideologie "das der Wirklichkeit fremd und metaphysisch-fetischisiert gegenüberstehende

2 An Franz Mehring schreibt Friedrich Engels 1893: „Die Ideologie ist ein Prozess, der zwar mit Bewusstsein vom so genannten Denker vollzogen wird, aber mit einem falschen Bewusstsein. Die eigentlichen Triebkräfte, die ihn bewegen, bleiben ihm unbekannt, sonst wäre es eben kein ideologischer Prozess. Er imaginiert sich also falsche resp. scheinbare Triebkräfte." (MEW 1958, Bd. 39, 97) 
idealistisch-verkehrte Bewußtsein, das die tatsächlichen Verhältnisse auf den Kopf stellt und in diesem Sinne verkehrt widerspiegelt" (Rauh 1969, 290, zit. nach Sorg 1981, 90).

Gemeinhin wird Ideologie mit "Ideenlehre" oder "Weltanschauung" übersetzt. Peter Christian Ludz (1981) bietet eine Definition, die er selbst als Substrat und relativ abstrakt bezeichnet. Sie entspricht der von Pelinka $(2004,176)$ als „bemüht wertfrei“ bezeichneten Variante, die im Gegensatz zum negativ gewerteten Ideologiebegriff in der Tradition von Marx und Engels steht. Ludz also fasst zusammen: „Ideologie ist eine aus einer historisch bedingten Primärerfahrung gespeiste, systemhafte und lehrhafte Kombination von symbolgeladenen theoretischen Annahmen, die spezifischen historisch-sozialen Gruppen ein intentional-utopisches, tendenziell geschlossenes und dadurch verzerrtes Bild von Mensch, Gesellschaft und Welt vermittelt und dieses Bild für eine bestimmte politisch-gesellschaftliche Aktivität bei strenger Freund-Feind-Polarisierung programmatisch-voluntaristisch organisiert." (Ludz 1981, 37)

Um Antworten auf die zweite Frage, worin genau die ideologische Funktion von Stereotypen besteht, bemühen sich nur wenige Autor*innen. Zu nennen sind die Linguistin Uta Quasthoff, der polnische Sprachphilosoph Adam Schaff und mit Stuart Hall einer der wichtigsten Vertreter der Cultural Studies, der sich u. a. in dem Beitrag Das Spektakel der Anderen (Hall 2004, 108-167) mit Stereotypisierung als Repräsentationspraxis beschäftigt hat.

Nach einer Erläuterung verschiedener Ideologiebegriffe gelangt Quasthoff zu dem Ergebnis, dass „die marxistische Theorie der auf ökonomischen Bedingungen beruhenden Klassengesellschaft [...] für die Problematik der Vorurteile den größten Erklärungswert hat" (Quasthoff 1973, 129). Danach würden Vorurteile und Stereotype als Herrschaftsmittel eingesetzt und dienten der Machtstabilisierung der herrschenden Klasse. Dennoch sei Differenzierung geboten: „Man wird einzelne soziale Vorurteile oder ,Stereotype' zu betrachten haben, wird fragen müssen, welcher Art von Ideologie sie zuzurechnen sind, welchen Grad von Realitätsgehalt, von Rationalität sie beinhalten, welche Formen gesellschaftlichen Handelns sie implizieren.“ (Quasthoff 1973, 148) „Wertfrei“ könnten diese Fragen sowie die nach den Funktionen von Vorurteilen nicht beantwortet werden, entscheidend sei die Ergründung des cui bono? und damit der Interessengebundenheit von Vorurteilen. 
Schaff sieht eine enge Verbindung zwischen Stereotypen und Ideologie. In „Rückkopplungsprozessen“ verstärkten sie sich gegenseitig: „Das Denken in Stereotypen unterstützt die Abwehrfunktion der Ideologie [gegenüber anderen Ideologien und ihren spezifischen Stereotypen, M.T.], und das ideologische Denken fördert die Genese und Fixierung der Stereotypen.“ (Schaff 1980, 99) So sei es „nicht weiter erstaunlich, daß die Stereotypen ein geradezu unersetzliches Instrument im Arsenal des politischen Kampfes sind" und folglich politische Funktionen erfüllen (ebd.). Neben der politischen und der Funktion der Ideologiebildung nennt Schaff die Abwehrfunktion und die sozialintegrative Funktion. Alle vier seien eng miteinander verbunden. „Der gemeinsame Nenner, auf den man diese Funktionen reduzieren kann, ist die Verhüllung der sozialen Wirklichkeit." (Schaff 1980, 102).

Stuart Hall ist in seiner Analyse von Stereotypen stark beeinflusst durch die Arbeiten Louis Althussers, der wiederum vom Marx'schen Ideologiebegriff und den Schriften Antonio Gramscis ausgegangen ist, sich aber teilweise vom Marx'schen Ideologiebegriff absetzt. ${ }^{3}$ Unter dem Eindruck der politischen Proteste von 1968 veröffentlichte Althusser 1970 den Aufsatz Idéologie et appareils idéologique d'Etat (Notes pour une recherche $)^{4}$, in dem er sich mit Institutionen („Apparaten“) innerhalb des kapitalistischen Systems und ihrer Macht, Ideologie zu verbreiten, beschäftigt. $\mathrm{Zu}$ den ideologischen Staatsapparaten, die die Existenz des repressiven Staatsapparates sichern, zählt Althusser Religion, Schule und Hochschule, Familie, Recht, Politik, korporatistische Verbände, Information und Kultur - sprich "die Medien“ - sowie Literatur, Kunst, Sport etc. (vgl. Althusser 1977, 119f.). Jene Apparate könnten aber, so der Autor, nicht vollständig von den Kapitalisten kontrolliert werden. Umso stärker sei ihr Bemühen, mittels Interpellation („Anrufung“) eine Subjektivierung zu erreichen, die aber nur eine scheinbare Handlungsfähigkeit, in Wirklichkeit

3 Althusser skizziert in Ideologie und ideologische Staatsapparate (1970/dt. 1977) u. a. den Entwurf einer „Theorie der Ideologie“ und gelangt zu einer Definition, wonach „Ideologie eine ,Vorstellung' des imaginären Verhältnisses der Individuen zu ihren realen Existenzbedingungen" $(1977,133)$ sei. Titel Ideologie und ideologische Staatsapparate (Anmerkungen für eine Untersuchung) erschienen. 
Unterwerfung und gerade nicht SUBJEKTIVIERUNG ${ }^{5}$ bedeute. Althusser erkennt in der Rede von der Handlungsfähigkeit und vom freien Willen zu bekämpfende Ideologie, weil gerade diese Anrufung die bestehenden ausbeuterischen Verhältnisse aufrechterhalte.

Diese Gedanken Althussers greift Stuart Hall auf. Denn wenn ideologische Staatsapparate als „Instanzen der Ausbildung und Vermittlung all dessen, was der Begriff der ,Kultur' umfasst" (Wolf 2004, 764f.), verstanden werden, ist ihre Analyse, konkret die Analyse von Sozialisationsinstanzen wie Familie, Schule, Religion, Medien, Sport, Militär etc. politisches Programm. Dabei sollte aber Abstand genommen werden von reduktionistischen, deterministischen, essentialistischen Betrachtungen, vielmehr der Zusammenhang zwischen Kultur und Kapitalismus gesehen werden, „wobei jede Seite dieses Zusammenhangs für die andere Ursache und Wirkung zugleich ist" (Wolf 2004, 765). Dies bezeichnet Althusser (2011/1962, 121f.) als „Überdeterminierung“. So gesehen ist Kultur Ursache wie Effekt von Klassenpositionen. Und so gesehen sind Cultural Studies immer auch ein politisches Projekt.

Mehr noch aber als Althussers Ausführungen zur Ideologie in dem Aufsatz Ideologie und ideologische Staatsapparate überzeugt Hall Althussers Annäherung an den Ideologie-Begriff, die jener in dem zuvor publizierten Aufsatz Für Marx (1968) vorgenommen hat. Darin beschreibe Althusser, so Hall $(2004,50)$, Ideologie als „Systeme der Repräsentation, bestehend aus Konzepten, Ideen, Mythen oder Bildern“ ${ }^{6}$ In diesen Systemen der

5 Althusser verdeutlicht durch die Unterscheidung zwischen Groß- und Kleinschreibung von ",Subjekt" bzw. "SUBJEKT“, was er unter der „,doppelten Spiegelstruktur der Ideologie“ versteht: „1. die Anrufung der ,Individuen' als Subjekte, 2. ihre Unterwerfung unter das SUBJEKT, 3. Die wechselseitge Wiedererkennung zwischen den Subjekten und dem SUBJEKT sowie der Subjekte untereinander und schließlich die Wiedererkennung des Subjekts durch sich selbst, 4. Die absolute Garantie, daß alles in Ordnung ist und daß alles gut gehen wird, solange die Subjekte nur wiederkennen, was sie sind, und sich dementsprechend verhalten: ,Amen!' [Hebräisch: ,Wahrlich, es geschehe!']." (Althusser 1977, 147f.)

6 Diese Beschreibung stammt allerdings tatsächlich mehr von Hall. Althusser hingegen weigert sich in Für Marx, Ideologie ausführlich zu definieren: „Es genügt, sehr schematisch zu wissen, dass eine Ideologie ein (seine eigene Logik und seine eigene Strenge besitzendes) System von Vorstellungen (Bildern, Mythen, Ideen oder Begriffen, je nachdem) ist, das im Schoß einer gegebenen Gesellschaft mit einer geschichtlichen Existenz und einer geschichtlichen Rolle begabt ist." (Althusser 1968, 181, auch 2011, 295) 
Repräsentation würden Menschen „ihre imaginären Beziehungen zu ihren wirklichen Existenzbedingungen leben“ (ebd.). "Leben“ steht für Hall in unmittelbarem Zusammenhang mit Erfahrung. „Durch die [Repräsentation, M. T.] und in den Systemen der Repräsentation der Kultur ,erfahren' wir die Welt: Erfahrung ist das Produkt unserer Verständnisfähigkeit, unserer Interpretationsraster. Folglich gibt es keine Erfahrung außerhalb der Kategorien von Repräsentation oder Ideologie." (Hall 2004, 52f.)

Repräsentationsforschung ist für Hall und andere Vertreter*innen der kritischen Cultural Studies dementsprechend Ideologieforschung bzw. in marxistischer Ausdrucksweise: Ideologiekritik. Er beschäftigt sich in Das Spektakel der Anderen (im Original The Spectacle of the "Other", 1997) mit ",Stereotypisierung" als Repräsentationspraxis“ und fragt: „Welche typischen Formen und Praktiken werden heute angewandt, um ,Differenz' in der Alltagskultur zu repräsentieren, und wo kommen diese populären Formen und Stereotypen her?" (Hall 2004, 108) Hall bietet am Ende seiner Analyse rassistischer Stereotype in populären Medien auch Antworten darauf, warum stereotypisiert wird und wer davon profitiert. Ohne offen „funktionalistisch" ${ }^{\prime 7} \mathrm{zu}$ argumentieren, wird die übergeordnete ideologische Funktion von Stereotypen durch die Nennung offensichtlicher Funktionen wie Reduzierung, Essentialisierung, Naturalisierung, Fixierung von Differenz, Inklusion und Exklusion, schließlich Machterhalt, sehr deutlich.

In diesem Abschnitt, in dem der Zusammenhang zwischen Stereotypen und Ideologie erläutert wurde, sind mit Franz Dröge, Adam Schaff, Uta Quasthoff, Karl Marx und Friedrich Engels, Louis Althusser und Stuart Hall einige Autor*innen zitiert worden, die in der deutschsprachigen Mainstream-Kommunikationswissenschaft nicht sehr häufig Erwähnung finden. Welche Autor*innen und welche Themen in einer Fachdisziplin präsent sind - etwa durch Publikationen und Zitationen - ist aus fachhistorischer Perspektive hochinteressant, ebenso, welche Forschungsgebiete betreten, welche Studien durchgeführt werden und welche nicht. Um den Stellenwert der Stereotypen- und Vorurteilsforschung in der deutsch-

7 Auch an Althussers Aufsatz wurde kritisiert, dass er „funktionalistische“ Aussagen enthalte (vgl. Bourdieu und Wacquant 1998, 132) und ökonomisch-deterministisch argumentiere - was Althusser in späteren Aufsätzen, z. B. in Elemente der Selbstkritik (1975) oder Anmerkung über die ideologischen Staatsapparate (1976) zu widerlegen versucht hat. 
sprachigen Kommunikationswissenschaft und damit auch um Wissenschaftler*innen, die Stereotypenforschung betrieben haben, geht es im folgenden Abschnitt, in dem ein auf Metaanalysen basierendes Phasenmodell vorgestellt wird.

\section{Phasen der Forschung zu Medien und Stereotypen}

Wissenschaftliche Konjunkturen, Theorieentwicklungen, Paradigmenwechsel und auch Begriffsbildungen finden nicht unabhängig von der sozialen Umwelt statt, in der Wissenschaft betrieben wird. Das gilt in besonderer Weise für die Sozialwissenschaften, deren Gegenstände sich aus gesellschaftlichem Wandel ergeben und deren Analyseergebnisse wiederum auf die weitere soziale Entwicklung einwirken können. In der Beschäftigung mit kommunikationswissenschaftlicher Stereotypenforschung vermischen sich verschiedene Perspektiven, eine sozialhistorische, eine fachhistorische sowie - mit der fachhistorischen eng verbunden - eine theorien- und ideengeschichtliche. Alle drei sind wichtig, um erklären zu können, warum es wann welche Art von kommunikations- und medienwissenschaftlicher Stereotypenforschung gegeben oder auch nicht gegeben hat oder warum es zu zeitlichen Verzögerungen im Vergleich zu anderen (nationalen) Wissenschaftskulturen gekommen ist.

In der deutschsprachigen Fachgeschichtsschreibung ist die Verbindung von Ideengestalt, Sozialgestalt und Milieu in Anlehnung an Dirk Kaeslers (1984) wissenschaftssoziologische Studie zur frühen deutschen Soziologie und ihren Entstehungsmilieus inzwischen recht verbreitet (vgl. Averbeck und Kutsch 2002; 2004; Meyen und Löblich 2006), freilich mit unterschiedlichen Schwerpunktsetzungen. So rücken entweder die Entwicklung und Akzeptanz von Ansätzen, Theorien und Methoden in den Mittelpunkt oder aber das Wissenschaftssystem oder die Akteur*innen, die als "Scharnier" (Meyen und Löblich 2006, 30) zwischen Ideengestalt und Sozialgestalt fungieren. Die deutschsprachige kommunikations- und medienwissenschaftliche Stereotypenforschung haben einige Wissenschaftler*innen in besonderer Weise geprägt, indem sie wie Gerhard Maletzke programmatische Aussagen getroffen und die Stereotypenforschung als dringend zu bearbeitendes Forschungsfeld bezeichnet haben (vgl. Maletzke 1963, 205; Maletzke 1966, 323) oder indem sie wie Franz Dröge (1967) oder Jörg Schweinitz (2006) Monographien zum Thema 
vorgelegt haben, die theoretisch wie methodologisch richtungsweisend waren und sind. Andere, wie etwa Franz Ronneberger, haben mehrfach die Gelegenheit genutzt, Studien zu Stereotypen in der Publizistik zu rezensieren und auf diese Weise bestimmte Ansätze in der Stereotypenforschung zu protegieren oder zu marginalisieren (vgl. Ronneberger 1987a; 1987b; 1988; 1989). Doch sind es nicht nur einzelne Wissenschaftler*innen, die durch ihre Arbeit einen wesentlichen Beitrag zur Stereotypenforschung leisten, sondern auch mehr oder weniger institutionalisierte und z. T. interdisziplinär zusammengesetzte Gruppen von Forscher*innen wie etwa am Duisburger Institut für Sprach- und Sozialforschung (DISS), die mediale Konstruktionen von Geschlecht, Ethnie, Alter etc. analysieren.

Ein weiteres Ziel fachhistorischer Forschung ist neben der Auseinandersetzung mit Ideen- und Sozialgestalt die Identifizierung von Phasen in der Entwicklung einer akademischen Disziplin. Für die verhältnismäßig junge Kommunikationswissenschaft liegen zahlreiche Überblicke, jedoch kaum Phasenmodelle vor. Lediglich Arnulf Kutsch und Stephanie Averbeck schlagen für den Zeitraum zwischen 1900 und 1960 eine Einteilung vor (vgl. Averbeck und Kutsch 2002; 2004), und auch Maria Löblich spricht von Phasen in der Entwicklung der Kommunikationswissenschaft hin zu einer empirischen Sozialwissenschaft (vgl. Löblich 2010, 62f.). In der US-amerikanischen sozialpsychologischen Stereotypen- und Vorurteilsforschung und speziell in der Rassismusforschung sind Phasenmodelle häufiger (vgl. Duckitt 2001; 2010; Dovidio et al. 2010), die z. B. Historical Shifts in Dominant Theoretical and Social Policy Approaches to Prejudice (Duckitt 2010, 31) benennen. Auch deutsche Forscher*innen beziehen sich darauf (vgl. Bornewasser und Waage 2006, 769) oder entwickeln wie Andreas Zick ein umfangreiches „Diskurs-Schema“ (vgl. Zick 1997, 17-24). Diese Überblicke und Phasenmodelle sind teilweise auf die kommunikationswissenschaftliche Stereotypenforschung übertragbar, "teilweise" deshalb, weil sich die genannten Autor*innen mit Rassismus auf einen Bereich innerhalb der Vorurteils- und Stereotypenforschung konzentrieren und weil sie - Zicks Studie ausgenommen - die US-amerikanische Forschung sowie die dortigen sozialpolitischen Trends in den Blick nehmen.

Inzwischen liegen aber auch für die Teildisziplin kommunikationswissenschaftliche Stereotypenforschung bzw. das transdisziplinäre Forschungsfeld Medien und Stereotype Daten vor, die es erlauben, Phasen der Stereotypenforschung zu benennen. Erhoben wurden sie mittels Metaanalysen: Zum einen wurden 119 Beiträge zu Stereotypen und 
Vorurteilen erfasst, die seit Gründung der beiden Fachzeitschriften Publizistik und Rundfunk und Fernsehen bzw. Medien $\mathcal{E}$ Kommunikationswissenschaft bis zum Jahr 2011 erschienen sind (= Metaanalyse I), zum anderen wurden Publikationen ausgewertet, die sich mit Medien und Stereotypen befassten, jedoch nicht ausschließlich von Kommunikationswissenschaftler*innen publiziert wurden (= Metaanalyse II). Das methodische Vorgehen und die Ergebnisse der Metaanalysen im Einzelnen sind ausführlich dokumentiert (vgl. Thiele 2015). Es geht in diesem Beitrag um die Phasen des Aufschwungs und der Marginalisierung kommunikationswissenschaftlicher Stereotypenforschung, die aufgrund der erhobenen Daten identifiziert werden konnten.

Die folgende Tabelle versammelt einige wichtige Daten zur Geschichte des 20. Jahrhunderts (Spalte 1), zur Fachgeschichte (Spalte 2) und zur Geschichte der kommunikations- und medienwissenschaftlichen Stereotypenforschung (Spalte 3). Dabei vermischen sich z. T. nationale und transnationale Perspektiven. Die Darstellung in Form einer Tabelle und die Nennung von Jahreszahlen könnten zudem dazu verleiten, von einer Chronologie der Ereignisse auszugehen. Aber gerade wenn es um Theorien und Methoden, die bereits erwähnte Verbindung von "Ideengestalt", "Sozialgestalt" und "Milieu“ geht (vgl. Kaesler 1984; Averbeck und Kutsch 2002), ist nicht von einer strikten Abfolge und eindeutigen Kausalitäten auszugehen. Deutlich werden soll vielmehr der Konnex zwischen sozialen und politischen Entwicklungen auf der einen Seite sowie Konjunkturen der Forschung zu Medien und Stereotypen auf der anderen Seite. 
Tab. 1: Forschung zu Medien und Stereotypen aus historischer Perspektive (Quelle: eigene Darstellung)

\begin{tabular}{|c|c|c|}
\hline Geschichte & Fachgeschichte & $\begin{array}{l}\text { Geschichte der kommunikations- } \\
\text { wissenschaftlichen Stereotypen- } \\
\text { forschung }\end{array}$ \\
\hline $\begin{array}{l}\text { Erster Weltkrieg } \\
1914-1918\end{array}$ & $\begin{array}{l}\text { Institutsgründung in } \\
\text { Leipzig } 1916\end{array}$ & \\
\hline $\begin{array}{l}\text { Weimarer Republik } \\
\text { Zwischenkriegszeit }\end{array}$ & $\begin{array}{l}\text { weitere Institutsgründun- } \\
\text { gen }\end{array}$ & $\begin{array}{l}\text { 1922 Lippmanns Public Opinion. } \\
\text { Es folgen empirische Studien von } \\
\text { Rice (1926), Katz \& Braly (1933) } \\
\text { u. a. }\end{array}$ \\
\hline $\begin{array}{l}1933 \text { NS-Macht- } \\
\text { übernahme } \\
\text { Verfolgung und } \\
\text { Emigration } \\
\text { Zweiter Weltkrieg } \\
\text { 1939-1945 und } \\
\text { Holocaust }\end{array}$ & $\begin{array}{l}\text { NS-konforme Zeitungs- } \\
\text { kunde } \\
\text { Emigration von Wissen- } \\
\text { schaftler*innen }\end{array}$ & $\begin{array}{l}\text { Aufschwung der US-Forschung. } \\
\text { In Deutschland gibt es keine } \\
\text { unabhängige publizistikwissen- } \\
\text { schaftliche Stereotypenforschung }\end{array}$ \\
\hline $\begin{array}{l}\text { Nach 1945: } \\
\text { Alliierte Besatzung } \\
\text { Kalter Krieg } \\
\text { Staatengründung/ } \\
\text { Teilung } \\
\text { Re-Education }\end{array}$ & $\begin{array}{l}\text { Institutsschließungen } \\
\text { Personelle Kontinuitäten } \\
\text { und Neuanfänge }\end{array}$ & $\begin{array}{l}\text { Stereotypenforschung findet in } \\
\text { der Publizistikwissenschaft nicht } \\
\text { statt }\end{array}$ \\
\hline $\begin{array}{l}\text { 1950er Jahre } \\
\text { „Wirtschaftswun- } \\
\text { der" } \\
\text { Beginn der Europäi- } \\
\text { schen Einigung }\end{array}$ & $\begin{array}{l}\text { Gründung von Rundfunk } \\
\text { und Fernsehen (1953) sowie } \\
\text { Publizistik (1956) }\end{array}$ & $\begin{array}{l}\text { In anderen Disziplinen wie der } \\
\text { Soziologie und Sozialpsychologie } \\
\text { wird zu Stereotypen und Vorur- } \\
\text { teilen geforscht. US-amerikani- } \\
\text { sche Studien als Vorbilder }\end{array}$ \\
\hline $\begin{array}{l}\text { 1960er Jahre } \\
\text { Große Koalition } \\
\text { "1968" }\end{array}$ & $\begin{array}{l}\text { Empfehlung des Wissen- } \\
\text { schaftsrats, Publizistik nur } \\
\text { an wenigen Standorten } \\
\text { anzubieten } \\
\text { Gründung der DGPuK } \\
\text { (zunächst DGPuZ) } \\
\text { Empirisch-sozialwissen- } \\
\text { schaftliche Ausrichtung } \\
\text { Theorien- und Methoden- } \\
\text { import }\end{array}$ & $\begin{array}{l}\text { Übersetzungen und Rezensionen } \\
\text { wichtiger Werke } \\
\text { Beginn der kommunikationswis- } \\
\text { senschaftlichen Stereotypenfor- } \\
\text { schung, z. B. in Münster }\end{array}$ \\
\hline
\end{tabular}




\begin{tabular}{|l|l|l|}
\hline $\begin{array}{l}\text { 1970er Jahre } \\
\begin{array}{l}\text { Zlub of Rome, Gren- } \\
\text { "Gastarbeiter" / } \\
\text { Migration } \\
\text { Neue soziale Bewe- } \\
\text { gungen }\end{array}\end{array}$ & $\begin{array}{l}\text { Aufbau, Ausbau, erweiter- } \\
\text { tes Themenspektrum } \\
\text { Medienwissenschaftliche } \\
\text { Studiengänge, Journalistik }\end{array}$ & $\begin{array}{l}\text { Theoretische und methodische } \\
\text { Weiterentwicklungen } \\
\text { Impulse aus anderen Disziplinen } \\
\text { Ausdifferenzierung, Untersu- } \\
\text { chung verschiedener Stereotyp- } \\
\text { arten }\end{array}$ \\
\hline $\begin{array}{l}\text { 1980er Jahre } \\
\text { 1982 "geistig-morali- } \\
\text { sche Wende } \\
\text { 1989 Mauerfall }\end{array}$ & $\begin{array}{l}\text { Weiter steigende } \\
\text { studentische Nachfrage } \\
\text { Konsolidierung }\end{array}$ & $\begin{array}{l}\text { Studien zu nationalen und } \\
\text { ethnischen Stereotypen, Ge- } \\
\text { schlechter- und Altersstereotypen }\end{array}$ \\
\hline $\begin{array}{l}\text { 1990er Jahre } \\
\text { Kriege (Golfkriege, } \\
\text { Jugoslawien, ...) } \\
\text { Migration } \\
\text { Europäisierung } \\
\text { Globalisierung }\end{array}$ & $\begin{array}{l}\text { Institutsneugründungen } \\
\text { Inhaltliche und methodi- } \\
\text { sche Diversifizierung }\end{array}$ & $\begin{array}{l}\text { Postmoderne Theorien } \\
\text { Realismus vs. Konstruktivismus } \\
\text { De-Konstruktion, De-Kategorisie- } \\
\text { rung } \\
\text { Cultural Studies }\end{array}$ \\
\hline $\begin{array}{l}\text { 21. Jahrhundert } \\
\text { "111" }\end{array}$ & $\begin{array}{l}\text { Internationalisierung } \\
\text { Umsetzung der „Bologna“- } \\
\text { Beschlüsse }\end{array}$ & $\begin{array}{l}\text { Intersektionale Ansätze } \\
\text { Religiöse Stereotype } \\
\text { Transdisziplinarität }\end{array}$ \\
\hline
\end{tabular}

Die Forschung zu Medien und Stereotypen setzt im deutschsprachigen Raum mit Verspätung ein: Erst in den 1960er Jahren werden erste Studien publiziert. In den USA hingegen sind bereits, häufig unter Beteiligung emigrierter Wissenschaftler*innen, wegweisende Arbeiten entstanden, die im deutschsprachigen Raum nur einige wenige Forscher*innen zur Kenntnis nehmen. Der Zeitraum zwischen 1945 und 1965 muss mit Blick auf die Publizistikwissenschaft als „Nullphase“ bezeichnet werden. Während in anderen Fächern, insbesondere der Soziologie und Sozialpsychologie, bereits beachtliche Forschungsanstrengungen unternommen werden, ist die westdeutsche Publizistikwissenschaft dazu noch nicht in der Lage.

Für den späten Start der kommunikationswissenschaftlichen Stereotypenforschung gibt es wissenschaftsexterne und -interne Gründe. Erst seit Mitte der 1960er Jahre, so Metaanalyse I, erscheinen Beiträge in Publizistik sowie Rundfunk und Fernsehen, in denen es um Stereotype und Medien geht. Dann aber ist vor allem aufgrund der Publikationen aus dem Münsteraner Institut ein erster Forschungsboom $\mathrm{zu}$ erkennen. Die 
Orientierung der Publizistikwissenschaft in Richtung empirische Sozialwissenschaft seit den 1960er Jahren scheint sich ebenso wie programmatische Aussagen und die gesellschaftliche Umbruchssituation, für die die Chiffre "1968“ steht, positiv auf den Beginn der kommunikationswissenschaftlichen Stereotypenforschung ausgewirkt zu haben. Doch zeigen sich alsbald auch einige Nachteile der Selbstdefinition der Disziplin als "moderne“ Sozialwissenschaft: Die Kommunikationswissenschaft ist weniger offen für Ansätze und Methoden, die als veraltet und überholt gelten, sie ist weniger offen für abweichende politische und theoretische Positionierungen. Das vertieft die Spaltung in Kommunikations- und Medienwissenschaft, die für die Forschung zu Medien und Stereotypen nicht ohne Folgen bleibt. Es setzt ein arbeitsteiliger Prozess ein, der dazu führt, dass unterschiedliche Forschungsgegenstände als relevant erachtet, Theorieansätze verfolgt und Methoden präferiert werden (vgl. Hickethier 2000; Schäfer 2000).

Insgesamt lässt sich für die 1970er und 1980er Jahre eine institutionelle und thematische Ausdifferenzierung feststellen. Es bilden sich neue Forschungsbereiche innerhalb geistes- und sozialwissenschaftlicher Fächer heraus, die wie z. B. die Imagologie oder die Interkulturelle Kommunikation auch mit Stereotypenforschung zu tun haben. Neben nationalen Stereotypen interessieren des Weiteren Geschlechter-, Alters- und, mit Bezug auf den Journalismus, auch Berufsstereotype (vgl. Fabris 1971; Weischenberg 1976). Die ersten Studien aber zu ,Gastarbeitern' (Delgado 1972), zur Darstellung der Frau im Fernsehen (Küchenhoff 1975) oder zu alten Menschen in der Werbung (Hastenteufel 1980) entstehen in anderen fachlichen Zusammenhängen. Die Kommunikationswissenschaft ist da Nachzüglerin; die Rezensionen in den Fachzeitschriften sind, gerade wenn es um Geschlechterstereotype geht, tendenziell negativ (vgl. Hackforth 1976; Ronneberger 1987a).

Einen Aufschwung nimmt die Forschung $\mathrm{zu}$ "Ausländerstereotypen" und "Rassismus in den Medien“ seit Ende der 1980er Jahre und verstärkt in den 1990er Jahren, als durch rassistisch motivierte Gewalttaten weltweit die Aufmerksamkeit auf das wiedervereinte Deutschland gelenkt ist. Das Sprechen über "Sich und ,die Anderen“" verändert sich, der "Dialog der Kulturen“ und "Integration" sind häufig verwendete Begriffe in kommunikationswissenschaftlichen Studien, die aber zugleich von der unhinterfragten Existenz dualistischer Konzepte zeugen. Erst die Diffusion postmoderner Theorien führt zur grundsätzlichen Infragestellung 
und De-Konstruktion sozialer Kategorien wie Nation und Ethnie, Religion, Geschlecht etc. Versucht wird, das Denken in Gegensätzen zu überwinden, wie sich $\mathrm{u}$. a. an der Transformation der Frauenforschung zu Gender- und Queer-Studies und an den zahlreichen Plädoyers für Transdisziplinarität, Transnationalität und Transkulturalität etc. ablesen lässt. So können vier Phasen der kommunikationswissenschaftlichen Forschung zu Medien und Stereotypen identifiziert und benannt werden:

\section{Vier Phasen der Forschung zu Medien und Stereotypen}

1. 1945-1965: die Nullphase

2. 1966-1974: die Startphase

3. 1975-1989: die Phase der Ausdifferenzierung

4. 1990 bis heute: die Phase der De-Konstruktion und Transformation

Abb. 1: Phasen der deutschsprachigen Forschung zu Medien und Stereotypen seit 1945 (Quelle: eigene Darstellung)

Phasen in der wissenschaftlichen Beschäftigung mit Medien und Stereotypen deuten auf veränderte theoretische Positionierungen, auf Paradigmenwechsel und "turns". Allerdings ist noch einmal zu betonen, dass diese Phasen nicht zwangsläufig als zeitlich aufeinander folgend verstanden werden sollten. Möglich sind immer auch parallele intra- und interdisziplinäre Entwicklungen und mehr oder weniger friedliche Koexistenzen wissenschaftstheoretischer Positionen. Auf sie möchte ich noch eingehen. Um aber nachvollziehbar zu machen, welche Gründe es für die Marginalisierung (ideologie-)kritischer Positionen in der deutschsprachigen Kommunikationswissenschaft gibt, wende ich mich abschließend dem Werk Franz Dröges zu, wohlwissend, dass er nur einer von gar nicht so wenigen Kommunikationswissenschaftler*innen ist, deren Forschung ab einem gewissen Zeitpunkt „out" war. 


\section{Wissenschaftstheoretische Positionen}

Explizite Bezüge zu Theorien finden sich in den meisten Studien zu Medien und Stereotypen nicht, weswegen der Vorwurf der Theorielosigkeit im Raum steht. Beklagt werden jedoch auch die verwirrende Vielfalt oder Beliebigkeit der theoretischen Bezugnahmen sowie ihre Abhängigkeit vom jeweiligen wissenschaftlichen Zeitgeist. Darüber hinaus gibt es in einigen neueren Studien Versuche, die verschiedenen Positionierungen innerhalb der kommunikations- und medienwissenschaftlichen Stereotypenforschung zu identifizieren (vgl. Pörksen 2000; Hafez 2002; Kessler 2009; Thiele 2015). Mit Verweis auf Winfried Schulz (1989), der zwischen "ptolemäischer und kopernikanischer Weltsicht" unterschieden hat, werden "realistische“ Positionen von "konstruktivistischen“ abgegrenzt. Doch versammeln sich unter dem Dach "konstruktivistische“ oder aber "realistische" Forschung" recht unterschiedliche Positionen. Bei den konstruktivistischen reichen sie von radikalkonstruktivistischen bis hin zu sozialkonstruktivistischen, bei den realistischen von normativen, ideologiekritischen bis hin zu positivistischen, funktionalistischen.

Die normative Perspektive erklärt sich aus den Anfängen der Stereotypen- und Vorurteilsforschung. Aufgrund der Erfahrungen mit Kriegen, Verfolgung, Massenmord und Propaganda in der ersten Hälfte des 20. Jahrhunderts entwickelte sich die Einstellungsforschung („attitudes“) und mit ihr die Stereotypen- und Vorurteilsforschung. Die Wissenschaftler*innen wollten verstehen, warum manche Menschen bereit sind, andere auszugrenzen und mit Gewalt und Vernichtung zu bedrohen. Stereotype erscheinen in dieser Perspektive als Fehlwahrnehmungen, als Urteilsverzerrungen, gar als Ergebnis fehlerhafter Denkprozesse, jedenfalls als etwas Inkorrektes (vgl. Lilli 1982). Die ideologiekritische Variante lautet, dass Stereotype Ausdruck eines „von oben“" und „durch die Medien“ induzierten falschen Bewusstseins sind. Dementsprechend scharf ist die Kritik an Ansätzen und Theorien, z. B. der Frustrations-Aggressions-Theorie oder dem Uses-and-Gratifications-Approach, in denen die Neigung $\mathrm{zu}$ Vorurteilen und Stereotypisierungen allein auf individuelle, innerpsychische Vorgänge oder auf spezifische Bedürfnisse zurückgeführt wird. Vielmehr sei, so der Politologe und Friedensforscher Fritz Vilmar, "von der grundlegenden geschichtlichen Prägung der politischen Aggressionspotentiale unserer Zeit durch reale materielle Lebensbedingungen und Herrschaftsverhältnisse" (Vilmar 1971, 363) auszugehen. 
Normative und ideologiekritische Ansätze sind in der Stereotypen- und Vorurteilsforschung weit verbreitet. Sie sehen sich aber auch fundamentaler Kritik ausgesetzt, die nicht nur politisch motiviert ist. Kritik richtet sich gegen die erkenntnistheoretischen Prämissen, die einen reflexiven und selbstkritischen Umgang mit Vorurteilen und Stereotypen verhindern würden. Eine Kritik stereotyper Einstellungen und Inhalte stehe schon vor der eigentlichen Analyse fest. Nahezu zwangsläufig ende normative Stereotypenforschung mit der Bestätigung stereotyper Muster und mit Appellen Stereotype abzubauen.

Gerade nicht normative, sondern wertfreie Wissenschaft zu betreiben, lautet dagegen das erklärte Ziel positivistischer Wissenschaft. Vorurteilsforschung solle frei von Vorurteilen sein. Eine Tendenz zu positivistischen und funktionalistischen Zugängen und Erklärungen zeichnet sich innerhalb der kommunikationswissenschaftlichen Stereotypenforschung nach der sogenannten kognitiven Wende in der Sozialpsychologie und der empirisch-sozialwissenschaftlichen Neuausrichtung in den 1960er Jahren ab. Positivismus und Funktionalismus stehen im Gegensatz zu linken, gesellschaftskritischen, normativen Ansätzen. Es gehe nicht darum, die Welt zu verändern und eine andere Gesellschaftsform zu etablieren, sondern um die neutrale und möglichst objektive Beschreibung dessen, was ist. Funktionale Stereotyp-Erklärungen setzen auf unterschiedlichen Ebenen an, vor allem auf der individuellen und der gesellschaftlichen (Mikro- und Makroperspektive). Einige Funktionen wie die der "Stabilisierung", "Orientierung" und "Integration“ werden als zentral betrachtet, auch eine "Ökonomie des Denkens" und die aus ihr folgende Tendenz zur „Komplexitätsreduktion“ dienen als Erklärung sowohl sozialer als auch individuell-psychischer Prozesse.

Positivistische und funktionalistische Ansätze werden ebenfalls scharf kritisiert, zunächst dafür, dass Stereotype, Vorurteile und Feindbilder als sozialpsychologische Notwendigkeiten gesehen werden. Unglaubwürdig und nicht erfüllbar erscheint der Anspruch, rational und objektiv Stereotype, ihre Funktionen und Dysfunktionen analysieren zu können. Letztlich würden mittels empirischer Forschung Rechtfertigungen für die Existenz und Stabilität von Stereotypen geliefert, wenn nicht gar gleich der Stereotypen-Begriff vermieden und von kognitiven Schemata gesprochen werde. Oder es folge dem rechtfertigenden Funktionskatalog am Ende doch noch der Aufruf zum Stereotypabbau. Schwer wiegt der Vorwurf der politischen Instrumentalisierbarkeit und Theorieferne: Die 
Orientierung an naturwissenschaftlichen Methoden und Denkweisen, die mit der sogenannten empirisch-sozialwissenschaftlichen Wende Einzug in die Kommunikationswissenschaft gehalten hat, habe nur eine vermeintliche "Neutralität" und „Wissenschaftlichkeit" befördert, stattdessen $\mathrm{zu}$ „Empirizismus" und einer gefährlichen Theorielosigkeit geführt, letztlich Stereotypenforschung verhindert.

Ein Vergleich der beiden Positionen ergibt deutliche Unterschiede im Wissenschaftsverständnis und konkret bei der Konzeption von Stereotyp, bei den in den Mittelpunkt gestellten positiven oder negativen Funktionen sowie der Einschätzung der Rolle und Verantwortung der Medien. Beide Positionen gehen jedoch davon aus, dass es möglich ist, einen Abgleich zwischen Medieninhalten und der Realität vorzunehmen. In beiden Fällen scheinen die Forschenden überzeugt, eher zu einem objektiveren Blick auf eine von ihnen unabhängig existierende Realität in der Lage zu sein als diejenigen, deren Vorurteile und Stereotype sie analysieren. Konstruktivistische Positionen stellen genau jenes „Außen-vor-Sein” und damit den Beobachterstandpunkt vermeintlich objektiver Forscher*innen in Frage. Diese Perspektive ist nun, wie hinlänglich von Konstruktivist*innen wie ihren Gegner*innen dargelegt wurde, nicht etwa neu, sondern reicht weit zurück in die Philosophiegeschichte. In Walter Lippmann einen frühen Konstruktivisten zu erkennen (vgl. Wilke 2007), mag da nicht weiter erstaunen. Erstaunlicher ist vielmehr, wie seine in Public Opinion versammelten Beobachtungen und Deutungen je nach politischer und wissenschaftlicher Interessenlage eingesetzt worden sind. Konstruktivistische Positionen interessieren sich jedenfalls weniger für den Realitätsgehalt medial vermittelter Botschaften als für ihr Potenzial, gesellschaftliche Realität bzw. das, was für gesellschaftliche Realität gehalten wird, herzustellen. Aus konstruktivistischer Sicht sind Diskussionen über den Realitätsgehalt von Stereotypen, Vorurteilen und Feindbildern müßig. Die sogenannte "kernel-of-truth-debate“ führe deshalb zu nichts, weil weder klar sei, was denn den Kern ausmache, noch was "die Wahrheit" ist.

So konkurrieren in der wissenschaftlichen Debatte über Stereotype sehr unterschiedliche (erkenntnis-)theoretische Positionen miteinander. Ihre Vertreter*innen sind je nach (wissenschafts-)politischer Situation mehr oder weniger marginalisiert. An einzelnen Personen und Karriereverläufen lässt sich das ebenso nachvollziehen wie an Zusammenschlüssen, Forscher*innengruppen, Netzwerken und Institutionen. Rückblickend hatten (ideologie-)kritische, ebenso wie stärker medien- und 
kulturwissenschaftlich orientierte Stereotypenforscher*innen in der deutschen Kommunikationswissenschaft einen eher schweren Stand. Die Fachgesellschaft DGPuK (Deutsche Gesellschaft für Publizistik- und Kommunikationswissenschaft) sowie die wichtigsten Fachzeitschriften dominierten empirisch-sozialwissenschaftlich orientierte Wissenschaftler*innen. Sie haben maßgeblich auch die Kriterien für qualitätsvolle Wissenschaft vorgegeben. Das zeigt sich selbst noch dort, wo es z. B. um Adornos Erben (Scheu 2012) geht und die Ausgrenzung kritischer Positionen mit Hilfe ebendieser, aus der Mainstream-Kommunikationswissenschaft stammenden Kriterien erklärt wird (vgl. Thiele 2015, 372).

Am Beispiel Franz Dröge lässt sich sehr gut nachvollziehen, welche Phasen des Aufschwungs und der Marginalisierung kommunikationswissenschaftlicher und gerade auch ideologiekritischer Stereotypenforschung es in der Kommunikationswissenschaft gegeben hat. Denn Dröges frühe Werke, seine Dissertation und Aufsätze, die Ende der 1960er Jahre publiziert und positiv rezensiert worden sind, fallen in die "Startphase" (vgl. Abb. 1) und stehen für einen Aufschwung der kommunikationswissenschaftlichen Stereotypenforschung. Mit seiner in den folgenden Jahren stattfindenden politischen Positionierung deutlich links von der Sozialdemokratie und seiner Hinwendung zu kultursoziologischen Fragestellungen beginnt jedoch die Marginalisierung seiner Person und seiner Perspektive im Fach. Ideologiekritische Stereotypen- und Vorurteilsforschung kommt erst wieder stärker mit der Etablierung der Cultural Studies im deutschsprachigen Raum zum Zuge. Um das nochmals zu verdeutlichen, möchte ich mit einigen Stellungnahmen zu Dröges Werk, vornehmlich seinen Studien zu Stereotypen, schließen.

\section{Marginalisierung}

Franz Dröge promoviert 1965 in Münster. Der Titel seiner Arbeit lautet Kommunikationsgewebe und Stereotypen. Terminologische und systematische Erwägungen zur funktionalen Publizistik. 1967 erscheint die Dissertation als Buch mit dem kürzeren und auf den Begriff Stereotyp verzichtenden Titel Publizistik und Vorurteil. Im Mittelpunkt stehen dennoch Stereotype; ihnen und ihrer Rolle im publizistischen Prozess ist die eine Hälfte der "ausdrücklich auf theoretische Fundierung angelegten Arbeit" (Prakke 1967, 7) gewidmet, die andere klärt einleitend "Grundbegriffe des 
publizistischen Systems“. Dröges Studie folgt der funktionalen Publizistik seines Doktorvaters Henk Prakke (vgl. Dröge 1967, 11). Unter Funktion versteht der Autor einen „dynamischen Beziehungsbegriff: Die Aussage wird auf den Kommunikator und den Rezipienten bezogen. Darüber hinaus beinhaltet der Begriff Leistung [Hervorhebung von M. T.] in zweifacher Hinsicht: einmal für die kommunizierenden Individuen, zum andere für das publizistische System, für eine Kollektivität“ (Dröge 1967, 227).

Gerhard Maletzke bespricht das Werk für Rundfunk und Fernsehen und geht auf die funktionale Publizistik ein. Mit ihr setze "sich in der Publizistikwissenschaft ein Ansatz immer mehr durch, [der] die ältere kausalistische Vorstellung eines einfachen Einwirkens der Aussage auf den Rezipienten“ (Maletzke 1968, 60) aufhebe. Stereotype stellten „eine der Variablen in diesem Prozess" (ebd.) dar. Die wissenschaftliche Beschäftigung mit ihnen hält Maletzke für dringend geboten (vgl. Maletzke 1963, 205; Maletzke 1966, 323). Er lobt Dröges „im Grenzgebiet zwischen Publizistik und Sozialpsychologie" (Maletzke 1968, 60) angesiedelte Forschung, seine "große Literaturkenntnis" und das "breite Detailwissen", findet aber seine "mit Fachbegriffen übermäßig angereicherte Sprache“ eher "anstrengend" (ebd.).

Hans Heinz Fabris, dessen Rezension zwei Jahre später in der Publizistik erscheint, wertet Dröges Arbeit ebenfalls als "gewichtigen Beitrag zur ,Richtungsdiskussion“" (Fabris 1970, 87) und zur Etablierung einer funktionalen Publizistik im Sinne Prakkes. Das große Verdienst der Studie liege darin, eine theoretische Basis für empirische Stereotypenforschung gelegt zu haben. Fabris benennt auch Aufgaben einer künftigen Stereotypenforschung: „Es wird vor allem zu untersuchen sein, in welchem Ausmaß bestehende Stereotypen durch die publizistischen Medien verstärkt werden und wo durch Nutzung des verbleibenden Spielraums zu deren Wandel beigetragen werden kann" (Fabris 1970, 86).

Der theoretischen Auseinandersetzung mit Stereotypen in Publizistik und Vorurteil folgen dann auch empirische Studien. So stellt Dröge 1968 in der Publizistik das Konzept einer empirischen Stereotypenforschung zur Analyse der Bild-Zeitung (Dröge 1968) vor, das neben methodischen Anregungen auf Ideologiekritik abzielt. Allmählich zeichnet sich eine Abkehr Dröges vom Funktionalismus Münsteraner Prägung und eine Hinwendung zu neomarxistischen Ansätzen ab. Offen Position bezieht Dröge 1973 im Vorwort der 2. Auflage des gemeinsam mit Rainer Weißenborn und Henning Haft herausgegebenen Bandes zu Wirkungen der Massenkommuni- 
kation. Nach einer Kritik an der bisherigen empirischen Kommunikationsforschung, welcher "Theorieunfähigkeit" und eine das herrschende politische System legitimierende Forschungspraxis vorgeworfen werden (vgl. Dröge 1969, XX), zeichnet der Verfasser den Gegenentwurf einer marxistischen, in der Kritik der Politischen Ökonomie gründenden Massenkommunikationstheorie, die „die ideologischen Zusammenhänge traditioneller Kommunikationstheorie“ (Dröge 1973, XXI) zu kritisieren habe. Auf empirische Forschung und den traditionellen Wirkungsbegriff müsse eine solche Theorie nicht verzichten, zeige sich doch in der Wirkung "die wirkliche Bestimmung der bürgerlichen Massenmedien, die sich aus dem Zirkulationsprozeß des Kapitals als dem wirklichen Bewegungssubjekt der bürgerlichen Gesellschaft ableiten lässt"(ebd.). Die Ausarbeitung einer kritischen Kommunikationstheorie erfolgt in Wissen ohne Bewußtsein. Materialien zur Medienanalyse der BRD (Dröge 1973), ein Werk, das Dröge gemeinsam mit Ilse Modelmog verfasst hat. Ziel ist, unter Berücksichtigung der Methoden historisch-materialistischer Gesellschaftsanalyse aktuelle medienökonomische Entwicklungen im „Spätkapitalismus“ zu kritisieren und „Bewusstsein“ zu schaffen.

Dröges politisches Bekenntnis bleibt nicht ohne Folgen für seine wissenschaftliche Karriere und auch für die kommunikationswissenschaftliche und vor allem ideologiekritische Stereotypenforschung im Fach. Dröge, der so lange erfolgreich innerhalb der Disziplin Publizistik- und Kommunikationswissenschaft ist, wie er als Schüler Henk Prakkes gesehen wird, hat durch die Positionierung links von der Sozialdemokratie sowie die Kritik an einem scheinbar unpolitischen Empirismus und Funktionalismus der Kommunikationswissenschaft seine Karrierechancen im Fach deutlich verringert. Nachfolger auf dem Lehrstuhl Prakkes in Münster wird Winfried B. Lerg, Dröge erhält aber 1972 einen Ruf an die Universität Bremen. Das Thema Stereotype verfolgt er dort nicht weiter, beschäftigt sich jedoch mit kultursoziologischen, öffentlichkeitstheoretischen, medienpädagogischen und -ästhetischen Fragen. Rückblickend erscheinen Publikationen wie der von Suhrkamp verlegte Band Die Kneipe. Zur Soziologie einer Kulturform oder "Zwei Halbe auf mich!“ (Dröge und Krämer-Badoni 1987) als Beleg dafür, dass Cultural Studies im deutschsprachigen Raum früher als gemeinhin angenommen betrieben worden sind.

In den späten 1970er und in den 1980er Jahren publiziert Dröge kaum mehr in Publizistik und Medien \& Kommunikationswissenschaft. Seine Bücher bleiben dort weitgehend unbeachtet, die wenigen Rezensionen sind 
eher negativ. 1998 aber werden in der Publizistik eine Zusammenstellung seiner wichtigsten Publikationen seit 1965 und eine Würdigung zum 60. Geburtstag veröffentlicht (vgl. Gebhardt 1998a; b). Hartwig Gebhardt zeichnet darin den wissenschaftlichen Weg Dröges nach und spricht die Folgen der politischen Positionierung seines akademischen Lehrers an: „Mit seinen Überlegungen - z. B.: unter den Bedingungen der Kapitallogik produzieren nicht nur Medien Ideologie, sondern auch Medienforscher - machte er sich allerdings im Fach nicht nur Freunde“" (Gebhardt 1998b, 78).

Andreas M. Scheu zählt 2012 Franz Dröge zu Adornos Erben, so der Titel seiner Studie zur Verdrängung kritischer Positionen aus der deutschen Kommunikationswissenschaft (vgl. Scheu 2012). Scheu, der sich unter Berufung auf Bourdieus Habitus-Konzept dem kommunikationswissenschaftlichen Feld und der Gruppe linker Akteure in diesem Feld nähert $^{8}$, spricht von einem teilweise auch selbstverschuldeten Verlust wissenschaftlichen, kulturellen und sozialen Kapitals, der letztlich zur Marginalisierung Dröges im Fach geführt habe (vgl. Scheu 2012, 188ff.). Denn, so Scheu über Dröge: „Er trug zu seiner randständigen Position im Fach auch selbst bei, indem er das Fach und seine Akteure bewusst provokant kritisierte und die Grenze zwischen inhaltlicher und persönlicher Kritik beziehungsweise moralischer Anklage teilweise überschritt [...]" (Scheu 2012, 197).

Eine etwas andere Sichtweise nimmt demgegenüber Gerd Kopper ein, der nicht nur Kollege und Ko-Autor Dröges, sondern ihm Zeit seines Lebens freundschaftlich verbunden war. In seinem Nachruf greift Kopper auch das von Dröge in die deutsche Kommunikationswissenschaft eingebrachte Thema Stereotype auf: „Die tragische Ironie des Wissenschaftlerlebens von Dröge besteht darin, dass seine Person die (auch heute noch) wirksamste und gröbste nachwirkende Stereotypisierung als neo-

8 Scheu bedient sich der von Bourdieu geprägten Begrifflichkeiten, um - wie ich finde mit Bourdieu, aber letztlich gegen Bourdieus eigentliche Intentionen die Ausgrenzung kritischer Positionen aus dem „wissenschaftlichen Feld“ zu erklären. Denn das „Kapital“", das Wissenschaftler wie Horst Holzer, Franz Dröge, Manfred Knoche oder Siegfried Weischenberg erwerben konnten, bemisst der Autor mit Hilfe von Kriterien (z. B. Zahl der Publikationen, Publikationsort, empirische Ausrichtung der Forschung, Teilnahme an Fachtagungen, „Zugehörigkeit zum engeren Kern des Faches“ vs. „Randständigkeit", , enger Kontakt zu den Akteuren der deutschen Kommunikationswissenschaft $\left.^{\prime \prime}\right)$, die aus der Mehrheitskommunikationswissenschaft stammen. 
marxistischer Dogmatiker im Wissenschaftsbetrieb der Publizistik- und Kommunikationswissenschaft erfahren hat." (Kopper 2002, 468) Kopper spricht damit etwas an, dessen sich Wissenschaftler*innen bewusst sein sollten: Auch Wissenschaft beruht auf Stereotypisierung, oder wie es Theodor W. Adorno und Max Horkheimer $(1998,206)$ formuliert haben: "Wissenschaft ist Wiederholung, verfeinert zu beobachteter Regelmäßigkeit, aufbewahrt in Stereotypen."

\section{Literatur}

Adorno, Theodor W., Else Frenkel-Brunswik, Daniel Levinson, und R. Nevitt Sanford. 1950. The Authoritarian Personality. New York, Evanston, London: Harper \& Row.

Adorno, Theodor W., und Max Horkheimer. 1998. „Elemente des Antisemitismus. Grenzen der Aufklärung". In Dialektik der Aufklärung. Philosophische Fragmente. Lizenzausgabe für die Wissenschaftliche Buchgesellschaft, 192-234. Darmstadt: Wissenschaftliche Buchgesellschaft.

Althusser, Louis. 1968. Für Marx. Aus dem Französischen von Karin Brachmann und Gabriele Sprigath. Frankfurt am Main: Suhrkamp.

Althusser, Louis. 1977. Ideologie und ideologische Staatsapparate. Aus dem Französischen von Rolf Löper, Klaus Riepe und Peter Schöttler. Hamburg/Westberlin: VSA.

Althusser, Louis. 2011 [1962]. „Widerspruch und Überdetermination. Anmerkungen für eine Untersuchung". In: Für Marx. Aus dem Französischen von Werner Nitsch u.a. Vollst. u. durchgesehene Ausgabe. Hrsg. und mit einem Nachwort von Frieder Wolf, 105-60. Frankfurt am Main: Suhrkamp.

Averbeck, Stefanie, und Arnulf Kutsch. 2002. „Thesen zur Geschichte der Zeitungs- und Publizistikwissenschaft 1900-1960“. Medien \& Zeit 17 (2/3): 57-67.

Averbeck, Stefanie, und Arnulf Kutsch. 2004. „Thesen zur Geschichte der Zeitungs- und Publizistikwissenschaft 1900-1960“. In Die Spirale des Schweigens. Zum Umgang mit der nationalsozialistischen Zeitungswissenschaft, herausgegeben von Wolfgang Duchkowitsch, Fritz Hausjell, und Bernd Semrad, 55-66. Wien: Lit.

Bornewasser, Manfred, und Marco Waage. 2006. „Rassismus“. In Handbuch der Sozialpsychologie und Kommunikationspsychologie, herausgegeben von Hans Werner Bierhoff, und Dieter Frey, 764-71. Göttingen: Hogrefe.

Bourdieu, Pierre, und Loï J. D. Wacquant. 1998. Reflexive Anthropologie. Übers. v. Hella Beister. Frankfurt am Main: Suhrkamp.

Delgado, Manuel J. 1972. Die "Gastarbeiter" in der Presse. Eine inhaltsanalytische Studie. Opladen: Leske.

Dovidio, John F., Miles Hewstone, Peter Glick, und Victoria M. Esses. 2010. „Prejudice, Stereotyping and Discrimination: Theoretical and Empirical Overview." In The Sage 
Handbook of Prejudice, Stereotyping and Discrimination, herausgegeben von Miles Hewstone, Peter Glick, und Victoria M. Esses, 3-28. Los Angeles: Sage.

Dröge, Franz. 1968. „Konzept einer empirischen Stereotypenforschung. Methodische Überlegungen zu einer Aussagen-Analyse der Bild-Zeitung“. Publizistik 13 (2/3): 340-47.

Dröge, Franz. 1970. „Der Funktionalismus in der Kommunikationswissenschaft. Henk Prakke zum 70. Geburtstag am 26. April 1970“. Publizistik 15 (1): 93-97.

Dröge, Franz. 1973. „Zur Kritik der publizistischen Wirkungsforschung. Anstelle eines Vorworts zur zweiten Auflage“. In Wirkungen der Massenkommunikation, herausgegeben von Franz Dröge, Rainer Weißenborn, und Henning Haft, Henning, 2. Aufl. 1973, IXXXVI. Frankfurt am Main: Athenäum Fischer.

Dröge, Franz. 1967. Publizistik und Vorurteil. Münster: Regensberg.

Dröge, Franz, und Thomas Krämer-Badoni. 1987. Die Kneipe. Zur Soziologie einer Kulturform oder "Zwei Halbe auf mich!“. Frankfurt am Main: Suhrkamp.

Dröge, Franz, Winfried B. Lerg, und Michael Schmolke. 1970. Publizisten zwischen Intuition und Gewißheit. Drei Vorträge. Assen: Van Gorcum \& Comp.

Dröge, Franz, Rainer Weißenborn, und Henning Haft. 1969. Wirkungen der Massenkommunikation, 1. Aufl. 1969. Münster: Regensberg.

Duckitt, John. 2001. „Reducing Prejudice: A Historical and Multi-Level Approach”. In Understanding Prejudice, Racism, and Social Conflict, herausgegeben von Martha Augoustinos, und Katherine J. Reynolds, 253-72. London: Sage.

Duckitt, John. 2010. „Historical Overview”. In The Sage Handbook of Prejudice, Stereotyping and Discrimination, herausgegeben von Miles Hewstone, Peter Glick, und Victoria M. Esses, 29-44. Los Angeles: Sage.

Eberhard, Fritz. 1964. „Erfahrungen mit Propaganda. Ein Mitgestalter der öffentlichen Meinung reflektiert". Die Zeit (48): 61, 27. November 1964.

Fabris, Hans Heinz. 1970. Rezension: Franz Dröge. 1970. Publizistik und Vorurteil. Publizistik 15 (1): 85-87.

Fabris, Hans Heinz. 1971. „Das Selbstbildnis des Kommunikators bei Tageszeitungen“. Publizistik 16 (4): 357-68.

Gebhardt, Hartwig. 1998a. „Bibliographie Franz Dröge. Eine Zusammenstellung seiner wichtigsten Publikationen 1965-1997 anläßlich seines 60. Geburtstages“. Publizistik 43 (1): 84-87.

Gebhardt, Hartwig. 1998b. „Personalien: Von der Publizistikwissenschaft zur Kultursoziologie. Franz Dröge zum 60. Geburtstag“. Publizistik 43 (1): 78-79.

Hackforth, Josef. 1976. Rezension: Erich Küchenhoff. 1975. Die Darstellung der Frau und die Behandlung von Frauenfragen im Fernsehen. Publizistik 21 (3): 378-81.

Hafez, Kai. 2002. Die politische Dimension der Auslandsberichterstattung. Bd. 1: Theoretische Grundlagen. Baden-Baden: Nomos.

Hall, Stuart. 2004. Ideologie, Identität, Repräsentation. Ausgewählte Schriften, Bd. 4. Hrsg. Juha Koivisto, und Andreas Merkens. Hamburg: Argument.

Hall, Stuart, Hrsg. 1997. Representation. Cultural Representations and Signifying Practices. London: Sage. 
Hall, Stuart, und Paul Du Gay, Hrsg. 1996. Questions of Cultural Identity. London: Sage.

Hastenteufel, Regina. 1980. Das Bild von Mann und Frau in der Werbung. Eine Inhaltsanalyse zur Geschlechtsspezifität der Menschendarstellung in der Anzeigenwerbung ausgewählter Zeitschriften unter besonderer Berücksichtigung des alten Menschen. Dissertation, Universität Bonn, o.V., Druck: Rheinische Friedrich-Wilhelms-Universität Bonn.

Hickethier, Knut. 2000. „Binnendifferenzierung oder Abspaltung - Zum Verhältnis von Medienwissenschaft und Germanistik. Das "Hamburger Modell' der Medienwissenschaft". In Über Bilder sprechen. Positionen und Perspektiven der Medienwissenschaft, herausgegeben von Heinz-B. Heller, Matthias Kraus, Thomas Meder, Karl Prümm, und Hartmut Winkler, 35-56. Marburg: Schüren.

Kaesler, Dirk. 1984. Die frühe deutsche Soziologie 1909 bis 1934 und ihre Entstehungs-Milieus. Eine wissenschaftssoziologische Untersuchung. Opladen: Westdeutscher Verlag.

Kessler, Eva-Marie. 2009. "Altersbilder in den Medien: Wirklichkeit oder Illusion?“ In Medien und höheres Lebensalter. Theorie - Forschung - Praxis, herausgegeben von Bernd Schorb, Anja Hartung, und Wolfgang Reißmann, 146-55. Wiesbaden: VS.

Kopper, Gerd. 2002. „Personalien: Franz Dröge gestorben“. Publizistik, 47 (4): 468-69.

Küchenhoff, Erich. 1975. Die Darstellung der Frau und die Behandlung von Frauenfragen im Fernsehen. Eine empirische Untersuchung einer Forschungsgruppe der Universität Münster. Stuttgart u.a.: Kohlhammer.

Lenk, Kurt. 1981. „Zum Strukturwandel politischer Ideologien im 19. und 20. Jahrhundert. Begriff und Phänomen des ideologischen Bewußtseins". In Ideologien im Bezugsfeld von Geschichte und Gesellschaft, herausgegeben von Anton Pelinka, 97-107. Innsbruck: InnVerlag.

Lippmann, Walter. 1922. Public Opinion. London: Allen and Unwin.

Lippmann, Walter. 1964. Die öffentliche Meinung. München: Rütten + Loening.

Löblich, Maria. 2010. Die empirisch-sozialwissenschaftliche Wende in der Publizistik- und Zeitungswissenschaft. Köln: Herbert von Halem.

Ludz, Peter Christian. 1981. „Entwurf einer Typologie des Ideologiebegriffs. Zu einer historisch-kritischen Ideologietheorie". In Ideologien im Bezugsfeld von Geschichte und Gesellschaft, herausgegeben von Anton Pelinka, 35-50. Innsbruck: Inn-Verlag.

Maletzke, Gerhard. 1963. Psychologie der Massenkommunikation. Theorie und Systematik. Hamburg: Verlag Hans-Bredow-Institut.

Maletzke, Gerhard. 1966. Interkulturelle Kommunikation und Publizistikwissenschaft. Publizistik 11 (3-4): 318-28.

Maletzke, Gerhard. 1968. Rezension: Franz Dröge. 1970. Publizistik und Vorurteil. Rundfunk und Fernsehen 16 (1): 60-62.

McQuail, Denis. 1994. Mass Communication Theory. Third Edition. London, Thousand Oaks, New Delhi: Sage.

MEW (1958) = Marx-Engels-Werke, Bd. 3, 13, 39 der deutschen Ausgabe, die auf dem vom Institut für Marxismus-Leninismus beim ZK der KPdSU besorgten 2. russ. Ausgabe fußt. Berlin (Ost): Dietz. 
Meyen, Michael, und Maria Löblich. 2006. Klassiker der Kommunikationswissenschaft. Fach- und Theoriegeschichte in Deutschland. Konstanz: UVK.

Ötsch, Walter Otto, und Silja Graupe, Hrsg. 2018. Walter Lippmann. Die Öffentliche Meinung. Wie sie entsteht und manipuliert wird. Frankfurt am Main: Westend.

Pelinka, Anton, Hrsg. 1981. Ideologien im Bezugsfeld von Geschichte und Gesellschaft. Innsbruck: Inn-Verlag.

Pelinka, Anton. 2004. Grundzüge der Politikwissenschaft. 2. überarb. u. erw. Aufl. Wien: Böhlau.

Pörksen, Bernhard. 2000. Die Konstruktion von Feindbildern. Zum Sprachgebrauch in neonazistischen Medien. Wiesbaden: Westdeutscher Verlag.

Prakke, Henk. 1967. Zum Geleit. In Franz W. Dröge. Publizistik und Vorurteil, 7-8. Münster: Regensberg.

Quasthoff, Uta. 1973. Soziales Vorurteil und Kommunikation. Eine sprachwissenschaftliche Analyse des Stereotyps. Ein interdisziplinärer Versuch im Bereich von Linguistik, Sozialwissenschaft und Psychologie. Frankfurt am Main: Athenäum.

Ronneberger, Franz. 1987a. Rezension: Christine Blumschein. 1986. Wie man(n) Frauen macht ... Das Fernsehen als Vermittler und Produzent von Geschlechterideologien. Publizistik 32 (2): 246.

Ronneberger, Franz. 1987b. Rezension: Helga Reimann, und Horst Reimann. 1987. Gastarbeiter: Analyse und Perspektiven eines sozialen Problems. Publizistik 32 (4): 563-64.

Ronneberger, Franz. 1988. Rezension: Georg Ruhrmann, und Jochem Kollmer. 1988. Ausländerberichterstattung in der Kommune. Inhaltsanalyse Bielefelder Tageszeitungen unter Berücksichtigung ,ausländerfeindlicher“ Alltagstheorien. Publizistik 33 (4): 708-9.

Ronneberger, Franz. 1989. Rezension: Roland Hannemann. 1987. Die Welt unterm Regenbogen. Massenkommunikation und Stereotypie am Beispiel der Unterhaltenden Wochenzeitschriften. Publizistik 34 (3): 387-9.

Schaff, Adam. 1980. Stereotypen und das menschliche Handeln. Wien, München, Zürich: Europaverlag.

Scheu, Andreas M. 2012. Adornos Erben in der Kommunikationswissenschaft. Eine Verdrängungsgeschichte? Köln: Herbert von Halem.

Schulz, Winfried. 1989. „Massenmedien und Realität. Die ,ptolemäische' und die ,kopernikanische' Auffassung". Kölner Zeitschrift für Soziologie und Sozialpsychologie, Sonderheft 30: 135-49, herausgegeben von Max Kaase, und Winfried Schulz.

Six, Bernd, und Iris Six-Materna. 2006. „Naive Theorien“. In Handbuch der Sozialpsychologie und Kommunikationspsychologie, herausgegeben von Hans-Werner Bierhoff, und Dieter Frey, 322-29. Göttingen: Hogrefe.

Sorg, Richard. 1981. „Die Ideologietheorie von Marx, Engels und Lenin. Zur materialistischen Analyse des Inhalts von Klassenbewußtsein“. Ideologien im Bezugsfeld von Geschichte und Gesellschaft, herausgegeben von Anton Pelinka, 79-94. Innsbruck: Inn-Verlag.

Thiele, Martina. 2015. Medien und Stereotype. Konturen eines Forschungsfeldes. Bielefeld: transcript. 
Thiele, Martina. 2017. „Kategorien, Stereotype, Intersektionalität”. In Von der Reflexion zur Dekonstruktion? Kategorien, Typen und Stereotype als Gegenstand junger Forschung. Beiträge zur zweiten under.docs-Fachtagung zu Kommunikation, herausgegeben von Barbara Metzler, Julia Himmelsbach, Diotima Bertel, Andreas Riedl, und Lara Möller. Unter Mitarbeit von Christian Berger, Paul Hahnenkamp, Barbara Klaus, und Michael Schwinghammer, 15-32. Wien: danzig \& unfried.

Thiele, Martina. 2019. Intersektionalität und Kommunikationsforschung: was ist mit Medien? In Feministische Theorie und kritische Medienkulturanalyse. Ausgangspunkte und Perspektiven, herausgegeben von Tanja Thomas, und Ulla Wischermann. Bielefeld: transcript, 2019 (in Druck)

Weischenberg, Siegfried. 1976. Funktionshierarchie und Auto-Stereotyp von Kommunikatoren. Beispiel Rundfunk-Sportjournalisten: Unterhalter fühlen sich nicht anerkannt. Rundfunk und Fernsehen 24 (4): 344-54.

Wilke, Jürgen. 2007. „Nicht nur ein Theoretiker der öffentlichen Meinung. Walter Lippmann Revisited”, (Reihe „Klassiker der Kommunikationswissenschaft heute“). Medien $\mathcal{E}$ Kommunikationswissenschaft 55 (4): 595-612.

Wolf, Rick. 2004. Ideologische Staatsapparate/repressiver Staatsapparat. In Historisch-kritisches Wörterbuch des Marxismus, Band 6.1: Hegemonie bis Ideologie, herausgegeben von Wolfgang Haug, Frigga Haus, und Peter Jehle, 761-72. Hamburg: Argument. http://www.inkrit.de/e_inkritpedia/e_maincode/doku.php?id=i:ideologische_staatsapparate_repressiver_staatsapparat. Zugegriffen: 16. November 2019.

Zick, Andreas. 1997. Vorurteile und Rassismus. Eine sozialpsychologische Analyse. Münster u.a.: Waxmann.

\section{Open Access}

Dieser Beitrag erscheint unter der Creative-Commons-Lizenz CC-BY-SA 4.0: https://creativecommons.org/licenses/by-sa/4.0/ 